\title{
Implementation of Intelligent Operation and Maintenance for Grid Dispatching and Control System
}

\author{
Jixiang Lu*, Liyan $\mathrm{Xu}$, Jun $\mathrm{Hu}$ and Jili Wang \\ Nari Group Corporation/State Grid Electric Power Research Institute, Nanjing, China \\ ${ }^{*}$ Corresponding author
}

\begin{abstract}
As the scale of the grid expanding, the operation and maintenance of power grid dispatching and control system becomes more complex. In order to deal with the challenge and improve the efficiency of operation and maintenance, the implementation of intelligent operation and maintenance for grid dispatching and control system is proposed. In this paper, some related technologies such as automatic fault locating and diagnosis, operation data visualization and DevOps deployment are studied as well. The new architecture can effectively improve the efficiency of operation and maintenance, and ensure the system operation safety and stability.
\end{abstract}

Keywords-intelligent operation and maintenance; grid dispatching and control system; fault locating and diagnosis; data visualization; DevOps

\section{INTRODUCTION}

The current power grid dispatching and control system still adopts the traditional method of operation and maintenance $(O \& M)$, it cannot meet the requirement of the grid scale expansion and proceeding vast O\&M data in time. Now 3 major issues come up: 1) Current O\&M work is complicated and most O\&M work are done manually in grid dispatching and control system, unified O\&M tool is unavailable so far, operators need to use different tools solely during the work. Fault detection is very difficult and it always involves massive effort from automation department of local State Grid, which is much inefficient; 2) Current O\&M work is short of data visualization presentation for multiple application processes, system resources, and equipment hardware status. Operators can only receive the warnings when the system fails. Without the overall picture of system O\&M, the issue fix mainly relies on technical on-site support personnel or remote telephone guideline of corresponding hardware and software suppliers, which stretches the problem-solving time; 3) The system software upgrade is based on the centralized software version control system, factory support staff bring the revised source code from software version control system to the site and recompile to generate new system software as well. The upgrade cycle time is very long and mistakes usually come out during the upgrading by accident due to human participation.

$\mathrm{Li}[1]$ studied visualization technology and integration technology, suggested the design of grid-wide information system centralization of business O\&M system. Zhang[2] analyzed security risks in current $\mathrm{O} \& \mathrm{M}$ system, and designed a link information management system based on the tree model, which is able to effectively manage the link information of security $\mathrm{O} \& \mathrm{M}$. $\mathrm{Wu}[3]$ proposed a technical framework suitable for the practical demand of the power system, try to pass $4 \mathrm{~A}$ (Account, Authentication, Authorization, Audit) standard, and strengthen the monitoring and audit on the existing system. Zhang[4] integrated two open-source monitoring software Ganglia and Nagios in a Hadoop open-source cloud computing platform, and uses a mobile message software FeiXin to achieve real-time monitoring of the cloud computing platform. Song[5] analyzed several key factors of combining intelligent monitoring technology with large data operation and maintenance, and proposed a theory of intelligent monitoring technology based on large data platform. Huang[6] analyzed the information system operation maintenance related technology, and put forward an intelligence operations system overall architecture. Chen[7] analyzed the status quo of the existing O\&M of automation system and the existing problems put forward to establish scientific and the necessity of intelligent electric power information O\&M system, and gave the key factors establishment of electric power dispatching automation system O\&M information, to provide theoretical guidance for the design of the electric power information management system for further research.

Therefore, it is urgent to design a new intelligent operation and maintenance system which implements O\&M intelligent monitoring with data visualization, comprehensive data analyzing, fault fast detecting and intelligent diagnosis. Online system software release and upgrade is also required.

The rest of the paper is organized as follows: an intelligent operation and maintenance framework is described in section II section III emphasizes some related key technologies in the design, including automatic fault locating and diagnosis, on line O\&M data visualization and DevOps deployment; section IV concludes the paper with the benefit and future work.

\section{FRAMEWORK}

The intelligent operation and maintenance architecture of grid dispatching and control system is designed based on data flow. The data, multiple level metrics, is collected from OS, middleware and applications. Collected data is cached and stored in distributed RRD-based database, then the data will be presented in visualization, and AI based detection tree method will be applied on fault detection, corresponding warnings will be generated on screen and send the notifications to the 
registered stakeholders by email or by short message. Figure I gives the details as below:

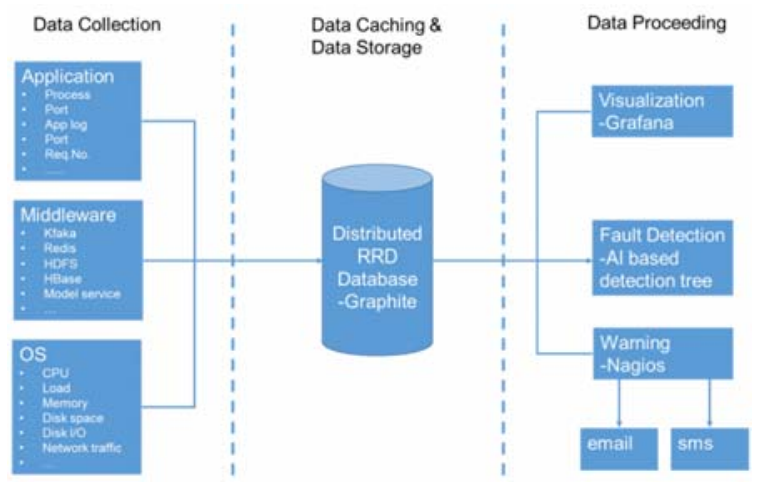

FIGURE I. DATA FLOW FOR GRID DISPATCHING AND CONTROL SYSTEM O\&M.

We focus on 3 level metrics: OS level metrics, such as CPU usage, memory usage and storage usage, etc. which indicates whether OS system is working correctly or not; middleware level metrics, which monitors the middleware such as Kafka, HBase, Redis, Message Bus and Service Bus, etc.; and application level metrics on application process, request number and port number, etc.

Usually bunch of metrics data will be sent to RRD database from time to time. To avoid the traffic and make the work efficiently, we cache the data by using Carbon-cache tool. Carbon-cache accepts metrics over various protocols and writes them to disk as efficiently as possible. This requires caching metric values in RAM as they are received, and flush them to disk on an interval using the underlying Whisper library. RRD storage is based on file, relatively easy and simple for the operation and maintenance. RRD is a circular database that provides a perfect solution for rough granularity storage, especially suitable for storing time series data. The size of the RRD file is fixed and it has a pointer to the latest data location, which is updated as the data is written.

Grafana is used for O\&M data visualization. It has the characteristics of dashboard and graphics editor, plentiful and flexible graphical options, etc. Nagios is used for O\&M data warning proceeding, corresponding mails or short messages will be generated and sent automatically. O\&M fault diagnose tree is applied in fault detection, we will give the details on next session.

\section{KEY TECHNOLOGIES}

In the architecture design of intelligent operation and maintenance for grid dispatching and control system, 3 key technologies will be applied: automatic fault locating and diagnosis, on line O\&M data visualization and DevOps deployment.

\section{A. Automatic Fault Locating and Diagnosis}

In the face of mass O\&M data in the grid dispatching and control system, it is necessary to take full account of the complex relationship between the time, space, application and other factors of the information. In the form of data visualization (thermo dynamic chart, multidimensional report, etc.), the operator can find the strong correlation between the indexes and diagnose the problem by combining with the empirical model of the previous problems. We get the experience of the operators and make it solidified into the monitoring system, it is to simulate the process of manual troubleshooting: checking the suspicious fault points, collecting the error, exception log, alarm and performance index of each module, organizing the base event data of fault locating, checking the corresponding pointes and handling the fault phenomenon. The fault diagnose tree structure can be formed through root cause analysis and consequence impact evaluation, the step-by-step exploration is carried out through a node in the tree.

In the fault locating, the whole fault diagnose tree is started from the beginning point of the fault phenomenon with inorder traversal method. The parameter output of the previous node is the parameter input of the latter one, check the existence of the fault root cause of this node, and obtain the evidence information of the corresponding phenomenon from various network elements respectively. The work is kept running until the leaf node is parsed. Then all the nodes satisfying the condition are returned according to the predefined weight. The diagnosis of the fault location is formed by synthesizing the results of each node after the end of traverse. Figure II gives details as below:

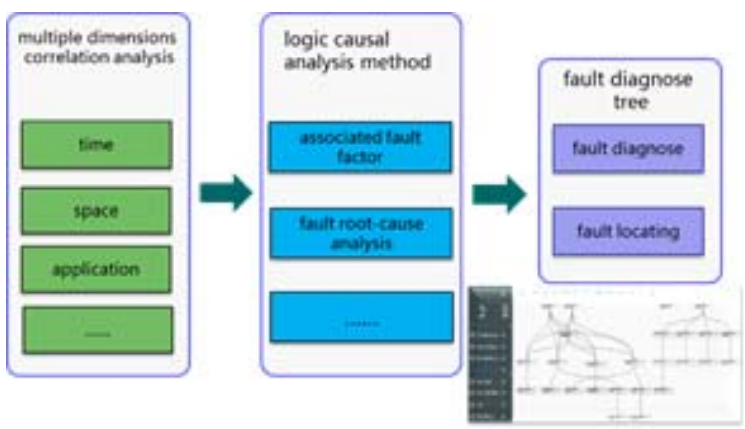

FIGURE II. AUTOMATIC FAULT LOCATING AND DIAGNOSIS AND SCREENSHOT

The intelligent fault root cause analysis is applied based on the analysis of the event-dependent multiple dimensions correlation, establishes the expert knowledge base based on historical fault location and processing experience set. It uses the theory and technology of AI, such as machine learning technology, makes the dynamic adjustment of the weight in the causality between multidimensional variables, and adjusts the weights of each checkpoint. The fault location method has more accurate error locating effect and more significant positioning efficiency than the previous traditional method.

We use Pearson to calculate the multiple dimensions correlation coefficient.

$$
r_{x y}=\frac{s_{x y}}{\sqrt{s_{x x}} \cdot \sqrt{s_{y y}}},\left|r_{x y}\right| \leq 1
$$

Equation (1) is Pearson correlation coefficient. $s_{x x}$ and $s_{y y}$ are variance, $s_{x y}$ is covariance. 


\section{B. Operations Data Visualization}

In daily system operation and maintenance, the operation data visualization offers a great deal in ensuring smoothness of operations and cost optimization. Excellent data visualization will improve O\&M personnel ability in understanding data, detecting issues, measuring risk, and increase confidence level of making necessary decision.

The operation and maintenance indicator data is retrieved from system log, middleware $\log$ and application $\log$ respectively. Then they are presented visually with Grafana tool. It helps in issue location and variation trend for system level health assessment as Figure III:

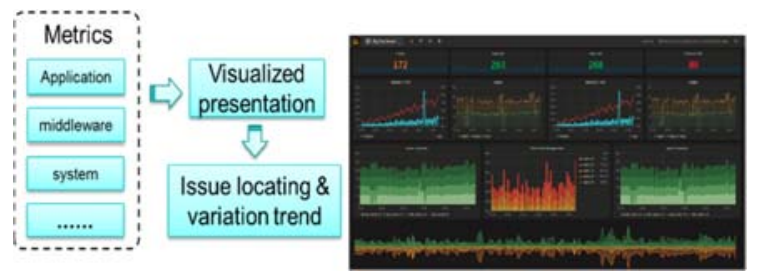

FIGURE III. OPERATION DATA VISUALIATION AND SCREENSHOT

After investigating data visualization tools on de-facto 5 criteria: comprehension, visual embellishment, design and aesthetics, visual metaphor and data memorability[8], we choose Grafana as the operation data visualization tool for the grid dispatching and control system. Grafana is an open source metric analytics \& visualization suite. It is most commonly used for visualizing time series data for infrastructure and application analytics. It has the characteristics of fully functional measurement dashboard and graphics editor, plentiful and flexible graphical options that can mix multiple styles. It contains a unique Graphite target parser to simplify the editing of metrics and functions. Grafana uses Flot technology to complete the fast rendering for client. Therefore, even if Grafana runs for a long time, it can be in good condition. So that users can create complex charts with smart axis formats. Figure III gives a screenshot of Grafana tool running on the O\&M system.

Here is the example of maintenance data analysis. Some customers in Beijing complain that they ran across the stuck of UI applications in grid control and dispatching system, they have to wait 2 3 seconds to get response from UI applications in some cases.

To figure out the root cause, we retrieve all UI logo in that 30-day timeframe. We use python script to filter other unnecessary information and only focus on startup cost time for each UI function, then find the top 10 UI functions which have large number in startup time bias. Each function will be marked with the top 3 abnormal timestamps. Based on the abnormal timestamp information, we can easily come up with the analysis result that some background operations make UI application performance down and stuck UI response at the corresponding timestamp. So developers will fix the root cause accordingly.

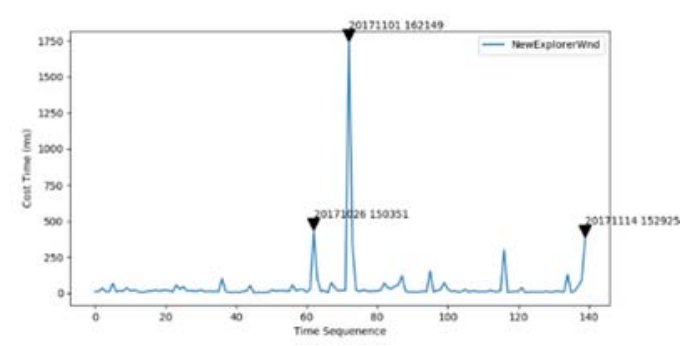

FIGURE IV. FUNCTION STARTUP COST TIME AND ABNORMAL TIMESTAMP

\section{DevOps Deployment}

System software upgrade work is an important part of operation and maintenance in the grid dispatching and control system from time to time. To make the work smoothly, we adopt DevOps deployment method since it is one of the best practices in software and service industry. DevOps is a crossdisciplinary community of practice dedicated to the study of building, evolving and operating rapidly-changing resilient systems at scale.

Taking into account the collaborative development that enables multiple distributed development teams to collaborate better with each other, DVCS (distributed version control systems) is used as the source code management tool. Each developer makes version update in a local code base on his/her own computer which does not need to be connected to the network. Code sharing among multiple developers is implemented through a remote central code repository by DVCS. In order to provide efficient support for the functional feature development, defect fix, software release and upgrade of grid dispatching and control system software, the branch management model of version control is applied and the software release and upgrade can be traced easily with DVCS.

At the same time, continuous integration and continuous deployment technology is introduced in the design. A continuous integration scheme based on remote central repository is proposed and it validates whether the system can pass build, code static checking and system smoke test after each code modification. The result will be sent to registered managers and corresponding developers by mail or short message. Versioning management covers source code, test code/scripts, database scripts, build and deploy scripts. It carries out continuous integration build and continuous deployment with virtualization technology and container technology if required. We standardize environment configurations across development, testing, and production which eliminate errors and hard-to-duplicate incidents caused by undocumented changes across environments[9]. Figure V gives the detail of DevOps deployment workflow as below: 


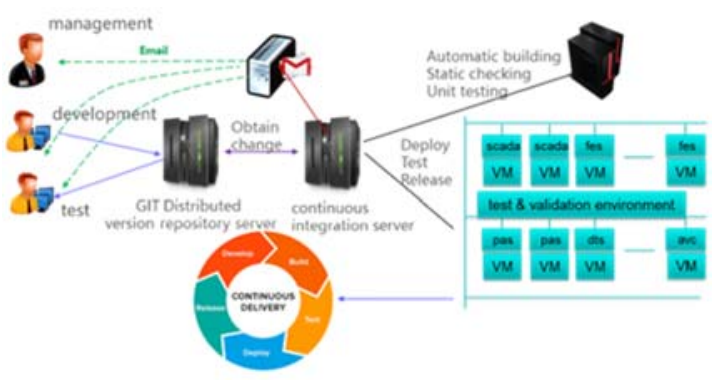

FIGURE V. WORKFLOW OF DEVOPS DEPLOYMENT

In order to solve the problem of software package dependencies and acquisition, a package management tool is proposed to manage platform software package. Based on source code versioning, it proposes to transform the software changes into a series of incremental and small-revised development principles, study and analyze the changes and dependencies of source code, and propose the method of generating incremental installation package to realize incremental upgrade automatically.

\section{SUMMARY}

With the vigorous development of China's smart grid, the number of the servers in the grid dispatching and control system is increasing rapidly, so the pressure of operation and maintenance keeps growing. When facing with vast amounts of operation and maintenance data, quickly locating the business level of problem, achieving precise alarm, and solving the problem in time become the general requirement of operation and maintenance.

In this paper, the problems and deficiencies of the current control system are analyzed. Combined with the current operational technology, an integrated intelligent operation and maintenance architecture is proposed. The mentioned key technologies, automatic fault locating and diagnosis, O\&M data visualization and DevOps deployment, are applied in O\&M system. It can effectively improve the automation and intelligent level of the operation and maintenance of the grid dispatching and control system, and implement active operation and maintenance to ensure the safety, stability and high efficiency of the system.

Built upon this work, future research could focus on multiple sites data center operation and maintenance, data security and performance optimization.

\section{ACKNOWLEDGMENT}

This work is financially supported by The National Key R\&D Program of China (No. 2017YFB0902600) and supported by State Key Laboratory of Smart Grid Protection and Control, partially supported by Collaborative Innovation Center of Novel Software Technology and Industrialization.

\section{REFERENCES}

[1] Li Yajie, Chen Zhenhua, Bai xiaojun.The design of the grid business operation and maintenance system[J].Automation \& Instrumentation, 2017(3):74-76.
[2] Zhang Xiao,Li Wei,Gao Minghui, et al. Tree Model Based Link Information Management for Power Monitoring System[J].Automation of Electric Power Systems,2016,40(11):126-131.

[3] Wu Zhengguo, Zhou Zhiguo. Investigation of Monitoring and Audit Technologies for Automatic Operation and Maintenance. Electronics Design \& Application. 2011, 38(10): 40-42.

[4] Zhang xiaoyin, Chen Guosheng. Intelligent Monitoring System on Cloud Computing Platform Based on Ganglia and Nagios. Journal of Anhui University of Science and Technology( Natural Science), 2016, 36(4): 69-74.

[5] Song Yueming,Xie Kejun. Research on Automatic Operation and Maintenance and Monitoring Technology Based on Large data Platform. Digital Technology \& Application. 2017, 6:102-103.

[6] Huang Jiaqi, Guo Zhigang, Sang Zixia, et al. Research on Intelligent Operation and Maintenance System Based on Power Information System Architecture. Telecom Power Technology. 2017, 34(2):113-114.

[7] Chen Zhenghua. Design of operations automation platform of state grid information system[J]. Industrial Instrumentation \& Automation, 2017 (2) :18-21.

[8] Nashriq Ramly, Noor \& Mat Nor, Fazli \& Ahmad, Nurul \& Haris Aziz, Mohd. (2012). Comparative Analysis on Data Visualization for Operations Dashboard. International Journal of Information and Education Technology. 287-290. 10.7763/IJIET.2012.V2.132.

[9] Microsoft DevOps TLP.pdf on https://www.microsoft.com/en-us/cloudplatform/development-operations. 